\title{
The ESO Precise RV Survey for Extra-solar Planets: Results from the First Five Years
}

\author{
M. Kürster \\ European Southern Observatory, Casilla 19001, Santiago, Chile
}

A. P. Hatzes and W. D. Cochran

Astronomy Dept., University of Texas, Austin, TX 78712-1083, USA

K. Dennerl and S. Döbereiner

Max-Planck-Institut für extraterrestrische Physik, D-85740 Garching, Germany

M. Endl ${ }^{1}$

European Southern Observatory, Casilla 19001, Santiago, Chile

\begin{abstract}
Results are presented from the first five years of the high precision RV survey carried out with the $1.4 \mathrm{~m}$ CAT+CES spectrograph at ESO La Silla. This RV survey of 37 solar-type stars was begun in Nov. 1992. Using an iodine gas absorption cell for self-calibration we currently achieve a long-term precision of $20 \mathrm{~m} / \mathrm{s}$ in a $30-\mathrm{min}$ exposure of a $5.5 \mathrm{mag}$ star. This value is the typical 'working' precision in survey work, i.e. an average over all observing conditions.

We present the RV data for our most variable star, $\iota$ Hor (G0V), with a possible periodicity of $600 \mathrm{~d}$ that could imply a companion of minimum mass $m \sin i=2.0 M_{\text {Jup }}$. However, these data are severely complicated by stellar activity to which a large part of the variability must be attributed. Our RV data for $\phi^{2}$ Pav (F8V) contain a possible period of $43 \mathrm{~d}$ which would imply a planet with $m \sin i=0.69 M_{\mathrm{Jup}}$. It must be stressed that for both stars the significance of the periods is still insufficient to conclude that planets are indeed orbiting them. We also show our data for a Hipparcos astrometric brown dwarf candidate, GJ 570A, that do not confirm expectations for a correspondingly high RV signal. Longer monitoring of the star is required in order to confirm a possible low amplitude variation.
\end{abstract}

\footnotetext{
${ }^{1}$ Also at: Institut für Astronomie, Univ. Wien, Türkenschanzstr. 17, A-1180 Wien, Austria
} 


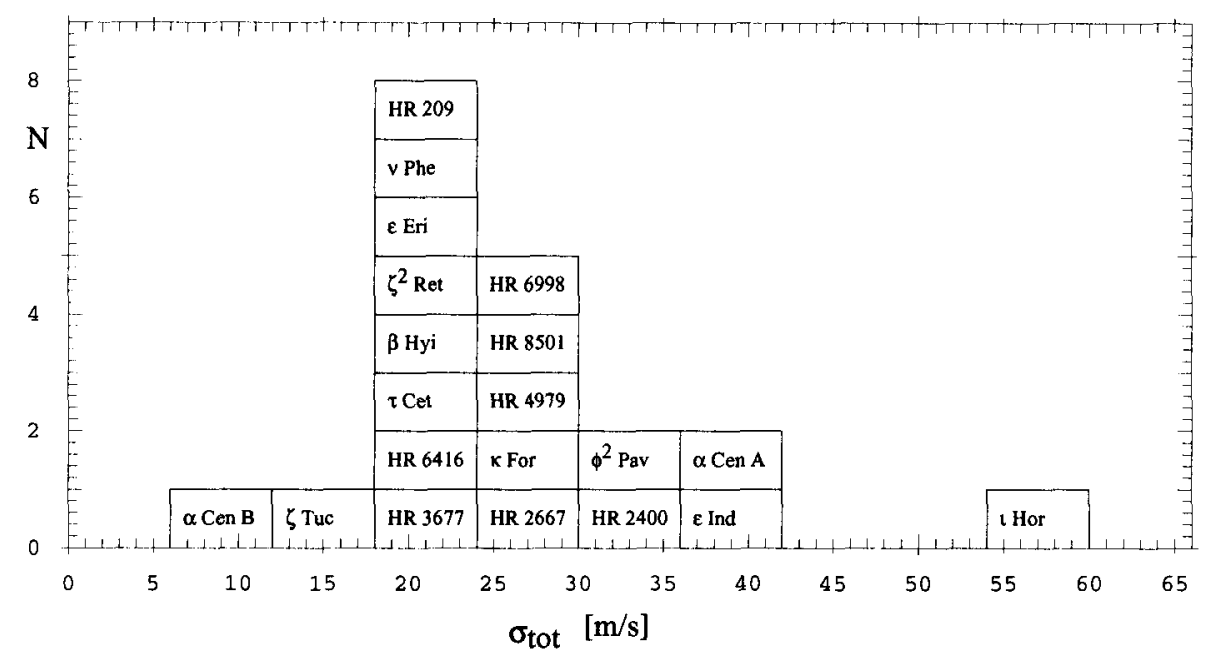

Figure 1. Histogram of total RV scatter for the 20 best observed stars in the ESO program.

\section{The ESO survey}

The ESO precise radial velocity survey was begun in November 1992 using the $1.4 \mathrm{~m} \mathrm{CAT}$ and the Long Camera of the CES spectrograph that provides a resolving power of $R=100000$. A low-pressure iodine gas absorption cell, temperature stabilized at $50^{\circ} \mathrm{C}$ is used to self-calibrate the data (Kürster et al. 1994). Only part of a single échelle order is recorded yielding a coverage of $\approx 48 \AA$ at a central wavelength of $5389 \AA$. A long-term precision of $20-25 \mathrm{~ms}^{-1}$ is achieved for stars brighter than $6 \mathrm{mag}$. The best case long-term precision is up to $10 \mathrm{~ms}^{-1}$ for brighter stars. Table 1 lists our program stars.

Fig. 1 shows a histogram of the total scatters of the differential radial velocities for our 20 best covered stars. As this histogram has not been corrected for observing conditions it includes data with varying signal-to-noise ratio. It thus reflects the "working conditions" in our survey work rather than our best case precision. Should there be genuine radial velocity variability in some stars an attempt to determine the measurement precision from this histogram alone would err on the side of overestimating the errors.

As we have previously shown (Hatzes et al. 1996) three of our stars, HR 2400, HR 3677 and $\kappa$ For, exhibit strong long-term variability in the form of linear trends for HR 2400 and HR 3677 and a slightly curved trend for $\kappa$ For. We can exclude substellar companions as the cause of this variation due to the large observed radial velocity range $\left(K \gg 500,950\right.$, and $2600 \mathrm{~ms}^{-1}$ for HR 2400 , HR 3677 and $\kappa$ For, respectively) and the high lower limit to the period $(P \gg 4 \mathrm{yr})$. The best explanation for the observed trends is that we have discovered three previously unknown $M$ dwarfs. Before including these three stars in the histogram (Fig. 1) the long-term trends were subtracted. 
Table 1. Program stars in the precise RV program at the ESO CES.

\begin{tabular}{cccccc} 
Star & V [mag] & Type & Star & V [mag] & Type \\
\hline$\zeta$ Tuc & 4.23 & F9V & $\beta$ Hyi & 2.80 & G2IV \\
HR 209 & 5.80 & G1V & $\nu$ Phe & 4.96 & F8V \\
HR 448 & 5.76 & G2IV & HR 506 & 5.52 & F9V \\
HR 753 & 5.82 & K3V & $\tau$ Cet & 3.50 & G8V \\
$\kappa$ For & 5.20 & G0Va & $\iota$ Hor & 5.41 & G0V \\
$\alpha$ For & 3.87 & F8V & $\zeta^{1}$ Ret & 5.54 & G3-5V \\
$\zeta^{2}$ Ret & 5.24 & G1V & $\epsilon$ Eri & 3.73 & K2V \\
$\delta$ Eri & 3.54 & K0+IV & $\alpha$ Men & 5.09 & G6V \\
HR 2400 & 5.60 & F8V & HR 2667 & 5.54 & G3V \\
HR 3259 & 5.98 & G7.5V & HR 3677 & 5.86 & G0V \\
GJ 433 & 9.79 & M2V & HR 4523 & 4.91 & G3V \\
HR 4979 & 4.85 & G3V & Prox Cen & 11.05 & dM6e \\
$\alpha$ Cen A & -0.01 & G2V & $\alpha$ Cen B & 1.33 & K1V \\
HR 5568 & 5.74 & K4V & HR 6416 & 5.48 & G8-K0V \\
Barnard & 9.54 & dM5 & HR 6998 & 5.86 & G4V \\
HR 7373 & 5.16 & G8IV & HR 7703 & 5.32 & K3V \\
$\phi^{2}$ Pav & 5.12 & F8V & HR 8323 & 5.58 & G0V \\
$\epsilon$ Ind & 4.69 & K4-5V & HR 8501 & 5.37 & G3V \\
HR 8883 & 5.64 & G4V & & &
\end{tabular}

\section{Data on individual stars}

\section{1. $\iota$ Hor $=$ HR 810}

The G0V star $\iota$ Hor (=HR 810) turns out to be a highly significant variable when compared with the sample (Fig. 1) via an F-test (Kürster et al. 1998). However, period analysis in the interval $2 \mathrm{~d}-4 \mathrm{yr}$ using the Lomb-Scargle algorithm (Scargle 1982) does not reveal a very significant period. The best period is near $600 \mathrm{~d}$, but its false alarm probability is still $>10 \%$. We estimate false alarm probabilities using a bootstrap randomization scheme as described by Murdoch et al. 1993, and Kürster et al. 1997. Our formal best-fit orbit for a companion to $\iota$ Hor yields $P=599 \mathrm{~d}, K=52.1 \mathrm{~m} / \mathrm{s}, e=0.49, m \sin i=2.0 M_{\mathrm{Jup}}$. Fig. 2 shows our RV data folded with the 599-day period together with the orbital fit. The residuals from this orbit are rather high, the r.m.s. scatter being $\sigma=42.3 \mathrm{~m} / \mathrm{s}$, i.e. still at the high end of the distribution in Fig. 1. Could there be a second signal in the data or does the variability have a different nature?

The rotation period of $\iota$ Hor was estimated by Saar \& Osten (1997) from CaIl data to be $P \approx 7.9 \mathrm{~d}$, while Saar et al. (1997) find $P \approx 8.6 \mathrm{~d}$. This makes $\iota$ Hor quite an active star. It is possible that the high scatter is largely due to line profile deformations induced by stellar activity such as star spots and variable granulation patterns (see Saar \& Donahue 1997). The best test to this hypothesis is to scrutinize the spectral region used for the RV measurements at very high resolving power searching for line bisector variations. We plan to use the new Very Long Camera of the CES that yields a resolving power of 220000 .

The absolute scatter of the RV data of $\iota$ Hor reaches almost the value expected for the estimated rotation period of the star (see Fig. 2 in Saar et al. 1998). If, however, the $600 \mathrm{~d}$ period is true, it is unlikely that it could be explained by stellar activity. Were it a rotation period, the implied stellar activity would be very low. On the other hand it is too short for the period of an activity cycle. 


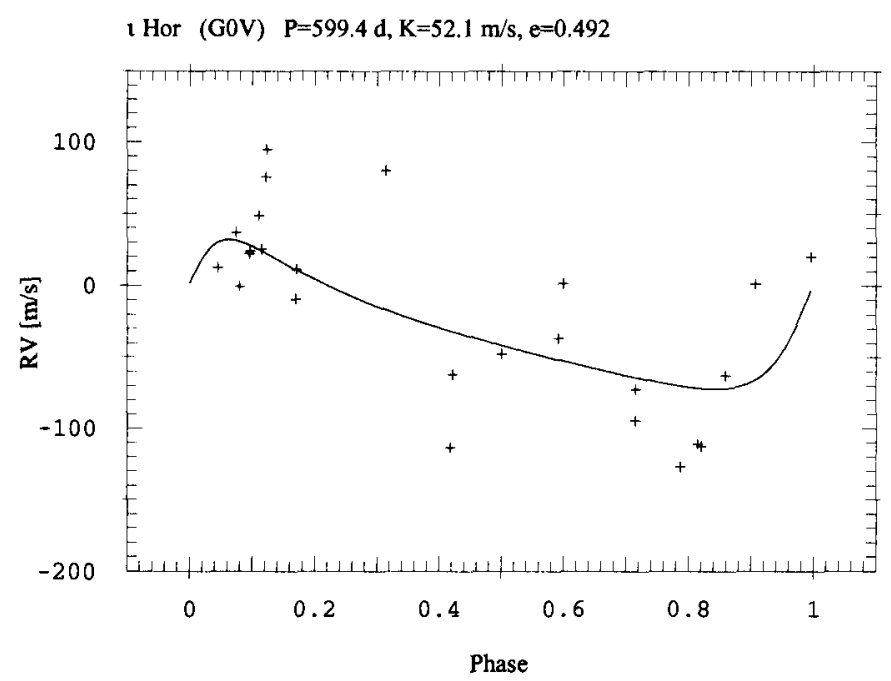

Figure 2. RV measurements for $\iota$ Hor folded with the best period.

At any rate, should the planetary signal be real but seen through activity induced scatter, several more of the $600 \mathrm{~d}$ cycles must be observed for a significant discovery.

\section{2. $\phi^{2} \mathrm{Pav}=$ HR 7875}

Our best candidate so far for having an orbiting planet (Kürster et al. 1998) is the F8V star $\phi^{2}$ Pav (=HR 7875). Period analysis in the interval $2 \mathrm{~d}-$ $4 \mathrm{yr}$ yields a best period of $42.46 \pm 0.10 \mathrm{~d}$ with a false alarm probability of 4 permille (or ' $2.9 \sigma^{\prime}$ ). The best-fit orbit is shown in Fig. 3 together with our RV measurements for $\phi^{2}$ Pav. The residuals from this fit scatter by $20.4 \mathrm{~ms}^{-1}$. The corresponding orbital parameters $K=39.5 \pm 6.0 \mathrm{~ms}^{-1}$ and $e=0.43 \pm 0.18$ imply a minimum companion mass of $m \sin i=0.69_{-0.15}^{+0.11} M_{\mathrm{Jup}}$ assuming a mass of $1.2 M_{\odot}$ for a main-sequence F8 star. The semi-major axis of this possible companion is $0.286 \pm 0.054 \mathrm{AU}$ which would thus orbit $\phi^{2} \mathrm{Pav}$ at about $3 / 4$ of the Sun-Mercury separation (0.387 AU).

The orbital parameters of the suspected companion to $\phi^{2}$ Pav would be strikingly similar to those of the planet around $\rho \operatorname{CrB}$ found by Noyes et al. (1997). Interestingly, a rather high eccentricity $(\approx 0.2)$ of the orbit of the planet around this star is suggested by recent measurements (Noyes et al., these proceedings).

In case the signal for $\phi^{2} \mathrm{Pav}$ is real we expect to be able to announce a significant discovery with some further measurements. In an attempt at improving our measurement precision by a factor $2-3$ we are currently implementing a treatment of the instrumental profile (IP) in the data modelling software. Should this improvement turn out to be achievable the significance of any real signal will be substantially increased. 


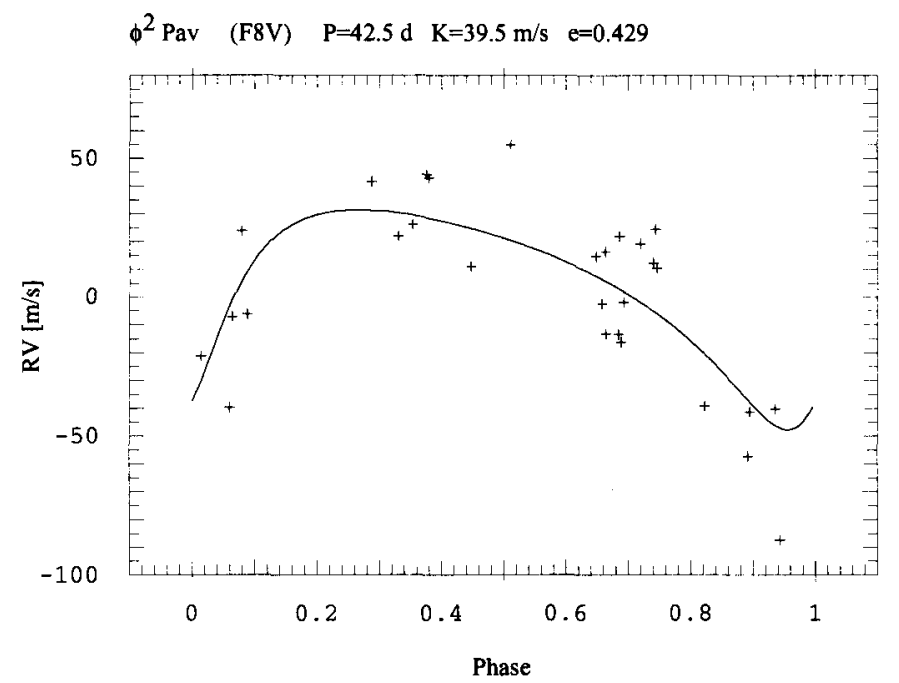

Figure 3. RV measurements for $\phi^{2}$ Pav folded with the best period.
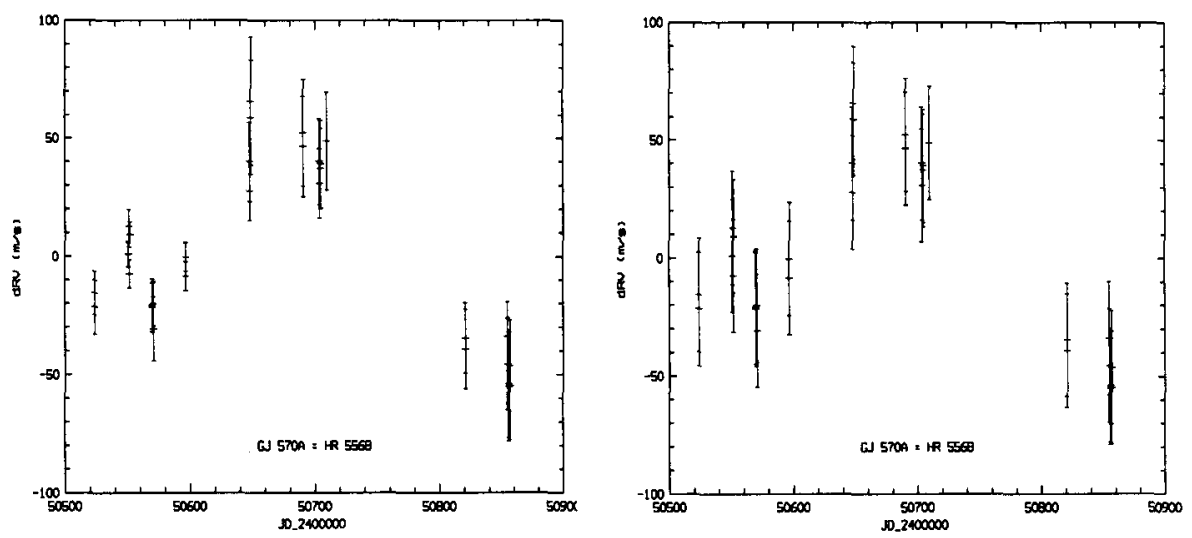

Figure 4. RV measurements for GJ 570A plotted vs. time. Left panel: The plotted error bars represent the variation among 7 different models applied to the data (see text). Right panel: The plotted error bars correspond to the median scatter in our sample of stars. 


\subsection{GJ 570A $=$ HR 5568}

To highlight the effect of a varying IP Fig. 4 shows our RV data for the K4Vstar GJ 570A (=HR 5568). Error bars plotted in the left panel represent the rms scatter among 7 different models applied to the RV data. In the modelling process each star+iodine spectrum is modelled using a pure iodine and a pure star spectrum. During 7 different epochs useful pure star spectra were obtained and used for a model together with the corresponding pure iodine spectrum. On average the differences between the models have an $\mathrm{rms}$ of $15 \mathrm{~ms}^{-1}$ representing the uncertainties that result from a varying IP. The right panel of Fig. 4 shows the same data with errors taken as the median scatter in our sample $\left(24 \mathrm{~ms}^{-1}\right.$; cf. Fig. 1), more representative of the overall error budget.

GJ $570 \mathrm{~A}$ is a Hipparcos astrometric variable (Bernstein \& Bastian, priv. comm.) and was recently included in our sample. The astrometric data point at the possible presence of a brown dwarf in a short period orbit $(<1$ year). Since the tentative astrometric solution would imply an almost edge-on orbit the implied RV variation would be of the order a few $\mathrm{kms}^{-1}$.

It is clear from the RV data in Fig. 4 that span about 1 year that such a high RV variability can be excluded. At best, some RV variability at much lower amplitude $\left(K \approx 50 \mathrm{~ms}^{-1}\right)$ might be present; if so, the corresponding period would be $>1$ year. However, we caution that it is certainly premature to draw this conclusion from the data set shown, and much longer monitoring of this object is required.

\section{Conclusions}

We have presented preliminary data from the ESO planet search program. Three stars, HR 2400, HR 3677 and $\kappa$ For, were found to exhibit long-period highamplitude trends indicating previously unknown M-dwarf companions.

Our most significant RV variable, $\iota$ Hor, is an active star, and it is not yet possible to decide whether the observed RV variability can be accounted for by stellar activity alone. Several more of the possible $600 \mathrm{~d}$ cycles most be observed.

The best candidate from our survey for having a planetary companion is $\phi^{2} \mathrm{Pav}$, however, with a false alarm probability still too high for the announcement of a significant discovery. If this star has a planetary companion with the tentatively derived orbital parameters, it bears interesting resemblance to the planet around $\rho \mathrm{CrB}$.

Our data set for the Hipparcos astrometric candidate GJ $570 \mathrm{~A}$ is not yet sufficient to deduce the presence of an orbiting planet. However, we can exclude the high RV variability predicted from the Hipparcos data. It therefore does not appear to have a brown dwarf companion in a short period orbit $(<1$ year).

Acknowledgments. This research was based on observations collected at the European Southern Observatory, La Silla. We are grateful to the ESO OPC for the generous allocation of observing time. WDC and APH acknowledge support by NASA Grant NAG5-4384. 


\section{Discussion}

Bernstein: Concerning Gliese 570A, astrometry has detected a peak at a few hundred days too. Here we have the ambiguity which is inherent to the astrometric techniques. So there might not necessarily be a contradiction.

Kürster: I agree as far as the period is concerned, especially as our period is not well established yet (not to mention that the variability itself is not totally significant). What we certainly do not find is the high radial-velocity amplitude that would be implied from the astrometric variation.

Hearnshaw: I am surprised that you observe at $R \sim 10^{5}$. A wider slit would give higher $S / N$ in a given exposure time, and thus give higher RV precision in spite of lower resolving power. Why do you therefore observe at $R \sim 10^{5}$ ?

Kürster: There are several reasons:

1. At $R \sim 10^{5}$ with the CAT+CES long camera (the one so far used) we already have a slit width of $1.2^{\prime \prime}$.

2. The RV precision depends on resolving power $R$, spectral bandwidth $B$ and signal in photons $S$ as

$$
\Delta \mathrm{RV} \sim R^{-3 / 2} B^{-1 / 2} S^{-1 / 2}
$$

as we know from simulations by Artie Hatzes. So $R$ is the most important parameter.

3. We will in the future work at $R=220000$ with the new CES long camera. A fiber link to the 3.6-m and image slicer will allow us to obtain this for an equivalent of $2.0^{\prime \prime}$ fiber aperture on the sky. We are not photon noise limited. This will put us in the situation to srutinize the data on our stars using exactly the same lines that are used to derive RVs in order to see intrinsic stellar variability that manifests itself in line shape variations of which the (presumed) RV variation is only a net effect. This important (indispensable) check cannot be done at $R<200000$.

Isaak: Your measurements on $\alpha$ Cen A and B suggest that you have an instrumental noise floor of the order of $10 \mathrm{~m} / \mathrm{s}$, as photon statistics would suggest a level of $1 \mathrm{~m} / \mathrm{s}$ for these bright objects.

Kürster: Your question obviously arises from the fact that I quoted a precision of $85 \mathrm{~m} / \mathrm{s}$ for an eleventh magnitude star (Proxima Centauri). At some point we are certainly limited by instrumental effects, the most important being an instrument vibration problem which we have recently improved considerably. Instrumental profile modelling will hopefully help us to tackle exactly this kind of effect.

Szécsény-Nagy: My question refers to the capabilities of the techniques you have just described. You presented precise data for $6 \mathrm{mag}$. to $11 \mathrm{mag}$. stars. Would you give an estimation for the case of an M-dwarf (possibly active) with visual apparent magnitude 16? What is the RV precision in this case (if any) and what is the exposure time needed? 
Kürster: Sixteenth magnitude is not feasible with our instruments, neither with the previous CAT+CES and long camera nor with the 3.6-m telescope plus CES and the very long camera. One would be read-out-noise limited. The useful limit is 11th magnitude.

\section{References}

Hatzes, A.P., Kürster, M., Cochran, W.D., Dennerl, K. \& Döbereiner, S., 1996, J. Geophys. Rev. (Planets) 101, 9285

Kürster, M., Hatzes, A.P., Cochran, W.D., Dennerl, K., Döbereiner, S., Endl, M., Vannier, M., 1998, Proc. Workshop Science with Gemini, 7-10 Dec. 1997, Florianopolis, Brazil, B. Barbuy (ed.), in press

Kürster, M., Hatzes, A.P., Cochran, W.D., Pulliam, C.E., Dennerl, K. \& Döbereiner, S., 1994, ESO Messenger, 76, 51

Kürster, M., Schmitt, J.H.M.M., Cutispoto, G. \& Dennerl, K., 1997, A\&A320, 831

Murdoch, K.A., Hearnshaw, J.B. \& Clark, M., 1993, ApJ413, 349

Noyes, R.W., Jha, S., Korzennik, S.G., Krockenberger, M., Nisenson, P., Brown, T.M., Kennelly, E.J. \& Horner, S.D., 1997, ApJ483, L111

Saar, S.H. \& Donahue, R.A., 1997, ApJ485, 319

Saar, S.H., Huovelin, R.A., Osten, R.A. \& Shcherbakov, A.G., 1997, A\&A326, 741

Saar, S.H. \& Osten, R.A., 1997, MNRAS284, 803

Saar, S.H., Butler, R.P. \& Marcy, G.W., ApJ498, L153

Scargle, J.D., 1982, ApJ263, 835 